\title{
Ocular arterial blood flow of choroidal melanoma eyes before and after stereotactic radiotherapy using Leksell gamma knife: 2 year follow up
}

Pia V Vécsei, Karl Kircher, Gunda Nagel, Sabine Toma-Bstaendig, Irene Ruhswurm, Michael Georgopoulos, Martin Zehetmayer

\begin{abstract}
Aims-To evaluate the effect of high dose stereotactic radiotherapy on the ocular blood flow of patients with uveal melanoma.

Methods-Colour Doppler imaging (CDI) was used to measure blood flow velocity and vascular resistance in the ophthalmic, short posterior, and central retinal arteries of nine patients suffering from uveal melanoma. The measurements were taken before, 6 months, 1 year, and 2 years after stereotactic radiotherapy. Irradiation was performed with the Leksell gamma knife with the 59 (41-66.5) Gy total marginal dose divided in two equal fractions. CDI results were compared with age and sex matched healthy control eyes.

Results-At each time of measurement, blood flow velocity in the central retinal artery of the affected eyes was significantly reduced whereas vascular resistance was only increased at the 2 year follow up. Blood flow velocity and vascular resistance in the short posterior arteries of melanoma eyes were also only significantly altered at the 2 year follow up. Blood flow velocity and vascular resistance in the ophthalmic artery of melanoma eyes were not changed at all follow ups.

Conclusions-In the melanoma eyes, blood flow velocity in the central retinal artery is reduced. High dose stereotactic radiotherapy with the Leksell gamma knife and a 59 (41-66.5) Gy total marginal dose in two fractions leads to a significant reduction of blood flow and a significant increase in resistance variables in the small ocular arteries within 2 years.

(Br f Ophthalmol 1999;83:1324-1328)
\end{abstract}

The colour Doppler sonography simultaneously produces a two dimensional grey scale B-image and a colour coded image of the blood flow recorded by a pulsed Doppler. ${ }^{12}$ This emitted impulse is reflected from the moving particles and may be examined according to its phase and frequency shift variances. The imaging subsequently describes a colour coded wave form (vessel) in the B-image based on the time cycle. The monitor shows blood flow areas as colour pixels while the simultaneously observed B-mode indicates the same area as an anatomical image.
High resolution and computerised technologies ${ }^{3}$ have been developed to detect even small vessels ${ }^{4}$ (for example, the central retinal artery) and to determine the circulatory direction, blood flow velocity (maximum, median, and minimum $)^{2}$ and resistance indices (RI, Pourcelot, ${ }^{5}$ pulsatility index, $\mathrm{PI}^{6}$ ). Colour Doppler assessment of the normal ocular blood flow has been reported by several authors. $^{7-9}$

Effective vasculature is essential for tumour growth. Many authors used the colour Doppler to measure tumour blood flow velocity in malignant melanomas of the uvea and to describe frequency shifts after therapy. ${ }^{10-12}$

We doubted the reliability and repeatability of the remeasurements because of tumour vessel direction, size, and the definition of an exact location over a period of time. We were interested in how far gamma knife radiosurgery would influence blood flow in the normal vasculature; this has not been reported before.

Uveal melanoma is the most common primary ocular malignancy. At our institution, the Leksell gamma knife stereotactic radiotherapy has become a treatment under investigation for selected patients with uveal melanoma. ${ }^{13}$

In performing colour Doppler examinations in a tumour vessel or tumour base vessel, possible inaccuracies are related to the facts that blood flow in these vessels is low and inconstant and that the direction of blood flow is often not identifiable. Furthermore, the angle between the vessel and the transducer often exceeds $60^{\circ}$, thus making the results inaccurate.

Defined measurement points are helpful for recording changes in ocular blood supply variables. To study effects after radiotherapy in our sample, blood flow velocities and resistance variables were evaluated in three unequivocal locations - in the ophthalmic artery, in the short posterior ciliary arteries, and in the central retinal artery.

This article describes the results of our investigations on nine patients with uveal melanoma after high dose stereotactic radiotherapy following a 2 year observation period. The small sample size was because of the long follow up time.

Patients and methods

A $128 \mathrm{XP} / 10$ Acuson (Erlangen, Germany) with a combined $7.0 \mathrm{MHz}$ ultrasound probe (linear array of 128 transducers) for the B-scan 
Table 1 Colour Doppler sonographic results of haemodynamically relevant variables in the ciliary artery

\begin{tabular}{|c|c|c|c|c|c|c|c|c|c|}
\hline & \multicolumn{3}{|l|}{$\operatorname{Vmax}_{P C A}$} & \multicolumn{3}{|l|}{$\operatorname{Vmin}_{P C A}$} & \multicolumn{3}{|l|}{$P I_{P C A}$} \\
\hline & Melanoma & Partner (P) & Control $(C)$ & Melanoma & Partner $(P)$ & Control (C) & Melanoma & Partner $(P)$ & Control (C) \\
\hline Before therapy (1) & $0.13(0.03)$ & $0.12(0.03)$ & $0.13(0.04)$ & $0.05(0.02)$ & $0.05(0.02)$ & $0.05(0.02)$ & $0.92(0.27)$ & $1.03(0.28)$ & $1.00(0.34)$ \\
\hline After 6 months (2) & $0.13(0.06)$ & $0.13(0.05)$ & - & $0.05(0.02)$ & $0.05(0.03)$ & - & $1.10(0.17)$ & $1.03(0.22)$ & - \\
\hline After 12 months (3) & $0.11(0.04)$ & $0.13(0.03)$ & - & $0.04(0.01)$ & $0.05(0.03)$ & - & $1.06(0.24)$ & $1.01(0.26)$ & - \\
\hline After 24 months (4) & $0.1(0.02)$ & $0.12(0.03)$ & - & $0.03(0.01)$ & $0.04(0.02)$ & - & $1.38(0.31)$ & $1.06(0.29)$ & - \\
\hline Statistically significant & \multicolumn{3}{|c|}{$\mathrm{C}$ to 1 : not significant $(\mathrm{p}=0.89)$} & \multicolumn{3}{|c|}{$\mathrm{C}$ to 1 : not significant $(\mathrm{p}=0.94)$} & \multicolumn{3}{|c|}{ C to 1 : not significant $(p=0.53)$} \\
\hline in Wilcoxon's test & \multicolumn{3}{|c|}{ C to 2 : not significant $(\mathrm{p}=0.91)$} & \multicolumn{3}{|c|}{$\mathrm{C}$ to 2 : not significant $(\mathrm{p}=0.73)$} & \multicolumn{3}{|c|}{$\mathrm{C}$ to 2 : not significant $(\mathrm{p}=0.44)$} \\
\hline$(\mathrm{p}=<0.05)$ & \multicolumn{3}{|c|}{ C to 3 : not significant $(p=0.22)$} & \multicolumn{3}{|c|}{ C to 3: not significant $(\mathrm{p}=0.16)$} & \multicolumn{3}{|c|}{$\mathrm{C}$ to 3 : not significant $(\mathrm{p}=0.67)$} \\
\hline & \multirow{2}{*}{\multicolumn{3}{|c|}{$\begin{array}{l}C \text { to } 4: \text { not significant }(p=0.08) \\
C \text { to } P 1 \text {. not significant }(p=0.52)\end{array}$}} & \multicolumn{3}{|c|}{$C$ to 4 : significant $(p=0.007)$} & \multicolumn{3}{|c|}{$C$ to 4 : significant $(p=0.013)$} \\
\hline & \multirow{2}{*}{\multicolumn{3}{|c|}{$\begin{array}{l}C \text { to } P 1 \text { : not significant }(p=0.52) \\
\text { C to } P 2 \text { : not significant }(p=0.70)\end{array}$}} & \multirow{2}{*}{\multicolumn{3}{|c|}{$\mathrm{C}$ to $\mathrm{P} 1$ : not significant $(\mathrm{p}=0.26)$}} & \multicolumn{3}{|c|}{ C to $P 1$ : not significant $(p=0.54)$} \\
\hline & & & & & & & \multirow{2}{*}{\multicolumn{3}{|c|}{$\begin{array}{l}C \text { to } P \text { 2: not significant }(p=0.78) \\
\text { C to P3: not significant }(p=1.0)\end{array}$}} \\
\hline & \multicolumn{3}{|c|}{$C$ to $P$ 3: not significant $(p=0.61)$} & \multicolumn{3}{|c|}{ C to P3: not significant $(p=1.0)$} & & & \\
\hline & \multicolumn{3}{|c|}{$\mathrm{C}$ to $\mathrm{P} 4$ : not significant $(\mathrm{p}=0.64)$} & \multicolumn{3}{|c|}{$\mathrm{C}$ to $\mathrm{P} 4$ : not significant $(\mathrm{p}=0.43)$} & \multicolumn{3}{|c|}{ C to $P$ 4: not significant $(p=0.66)$} \\
\hline
\end{tabular}

(maximum intensity $88 \mathrm{~mW} / \mathrm{cm}^{2}$ ) and 5.0 $\mathrm{MHz}$ for the pulsed Doppler (maximum intensity $39 \mathrm{~mW} / \mathrm{cm}^{2}$ ) was used for the blood flow measurements. In this study, $40 \mathrm{~mW} / \mathrm{cm}^{2}$ were applied for the B-scan and $25 \mathrm{~mW} / \mathrm{cm}^{2}$ for the pulsed Doppler. Penetration depth was set at $45 \mathrm{~mm}$.

The probe was applied to the patient in a supine position, eyes closed with no added pressure to the transducer. Methylcellulose $2 \%$ was used as coupling medium. With this routinely used technique we are able to produce a flawless image of the ophthalmic artery (OA), the central retinal artery (CRA), and the short posterior ciliary artery (PCA). The OA was measured just before crossing and lateral to the optic nerve. A PCA was measured as closely as possible to the optic nerve but as far away as possible from the tumour in order not to confuse the signal with the melanoma's feeder vessels and to avoid a possible steal phenomenon. The CRA was measured in the optic disc.

Angle correction was made according to the colour pixel flow direction when measuring all vessels. Whenever the angle exceeded $60^{\circ}$, it caused a lot of noise in the velocity measurements, and therefore no measurements were recorded. The maximum range of angle correction was set at $45^{\circ}$. The gate for the colour Doppler used for volume documentation and calculation was set at a minimum $(1 \times 1 \mathrm{~mm})$.

Each Doppler signal from each vessel was documented on video tape and evaluated with the calculation program provided in the system. The following values were determined: maximal (systolic) and minimal (end diastolic) blood flow velocity in the above mentioned vessels, the resistance index (RI) according to Pourcelot $^{5}$ and the pulsatility index (PI) according to Gosling and King. ${ }^{6}$ A comparison of the PI values merited special attention with regard to the total stability of the vessel resistance. The PI was calculated with the formula:

Pulsatility index $(P I)=\frac{\text { Vmax }-V m i n}{\text { Vmean }}$

Gamma knife radiotherapy is based on the use of a cross fire technique with 201 cobalt- 60 sources. ${ }^{14}$ Instrumentation, procedure of stereotactic tumour volume delineation and subsequent irradiation, ${ }^{14}$ modification for ocular treatment, ${ }^{15}$ as well as our preliminary results have been published previously. ${ }^{16}$
Since 1993, a total of 69 patients have been treated with gamma knife stereotactic irradiation. All consecutive patients from February 1995 to October 1995 were included in the Doppler study. Seven patients (three men and four women) presented with unilateral choroidal melanoma of the posterior pole and two patients (two men) with unilateral ciliary body melanoma. The patients' mean age was 63.6 (range $42-82$ years). The mean pretreatment tumour thickness of 6.8 (SD 2.5) $\mathrm{mm}$ was measured by using standardised echography (4.1-11.4 mm).

In seven patients the central tumour margin was within $3 \mathrm{~mm}$ from the optic disc or fovea. Taking all patients into account, the central tumour margin was within 3.2 (3.2) $\mathrm{mm}$ from the optic disc or fovea.

Patients were irradiated with 59 Gy marginal dose, ranging from 45 to 66.5 Gy marginal dose. The optic disc was irradiated with a mean of 26.6 (11.7) Gy, ranging from 5.6 to 45.8 Gy.

The colour Doppler examinations were performed by the same ophthalmologist and taken before, 6 months, 1 year, and 2 years after gamma knife treatment. The results of the colour Doppler were compared with the control group. The control group consisted of 18 healthy volunteers (18 eyes) with a mean age of 64.6 (range $44-83$ years).

We compared patients with a control group who were measured only once for the reason that repeatability and agreement on colour Doppler measurements have been found to be reliable according to the Harris's study group. ${ }^{17}$ So we could assume that the measurements from an age and sex matched healthy control population would change only very slightly over a period of 2 years, as long as the control group remains healthy.

We found it unethical to take choroidal melanoma patients as controls and not treat them for 2 years.

Only one eye per healthy volunteer was evaluated. The data are shown with average values (SD).

Informed consent was prerequisite from both patients and healthy volunteers.

The Wilcoxon rank sum test was applied to compare group values. Calculations were made by using the SAS statistic software package. A value of $\mathrm{p}<0.05$ was considered statistically significant. 
Table 2 Colour Doppler sonographic results of haemodynamically relevant variables in the central retinal artery

\begin{tabular}{|c|c|c|c|c|c|c|c|c|c|}
\hline & \multicolumn{3}{|l|}{$\operatorname{Vmax}_{C R A}$} & \multicolumn{3}{|l|}{$\operatorname{Vmin}_{C R A}$} & \multicolumn{3}{|l|}{$P I_{C R A}$} \\
\hline & Melanoma & Partner $(P)$ & Control $(C)$ & Melanoma & Partner $(P)$ & Control (C) & Melanoma & Partner $(P)$ & Control $(C)$ \\
\hline Before therapy (1) & $0.11(0.02)$ & $0.12(0.03)$ & $0.14(0.04)$ & $0.03(0.01)$ & $0.04(0.01)$ & $0.05(0.020$ & $1.23(0.23)$ & $1.10(0.32)$ & $1.25(0.32)$ \\
\hline After 6 months (2) & $0.08(0.02)$ & $0.14(0.04)$ & - & $0.03(0.01)$ & $0.04(0.02)$ & - & $1.14(0.15)$ & $1.22(0.25)$ & - \\
\hline After 12 months (3) & $0.08(0.02)$ & $0.12(0.04)$ & - & $0.02(0.01)$ & $0.04(0.01)$ & - & $1.35(0.28)$ & $1.14(0.16)$ & - \\
\hline After 24 months (4) & $0.05(0.20)$ & $0.13(0.05)$ & - & $0.01(0.01)$ & $0.04(0.02)$ & - & $1.70(0.32)$ & $1.25(0.20)$ & - \\
\hline Statistically significant & \multicolumn{3}{|c|}{$C$ to 1 : significant $(p=0.015)$} & \multicolumn{3}{|c|}{$\mathrm{C}$ to 1 : not significant $(\mathrm{p}=0.13)$} & \multicolumn{3}{|c|}{ C to 1 : not significant $(p=0.88)$} \\
\hline in Wilcoxon's test & \multicolumn{3}{|c|}{$C$ to 2 : significant $(p=0.0006)$} & \multicolumn{3}{|c|}{$\mathrm{C}$ to 2: significant $(\mathrm{p}=0.03)$} & \multicolumn{3}{|c|}{$\mathrm{C}$ to 2 : not significant $(\mathrm{p}=0.37)$} \\
\hline$(\mathrm{p}=<0.05)$ & \multicolumn{3}{|c|}{ C to 3: significant $(p=0.0003)$} & \multicolumn{3}{|c|}{$C$ to 3: significant $(p=0.01)$} & \multicolumn{3}{|c|}{$\mathrm{C}$ to 3 : not significant $(\mathrm{p}=0.44)$} \\
\hline & \multicolumn{3}{|c|}{$C$ to 4: significant $(p=0.0005)$} & \multicolumn{3}{|c|}{ C to 4: significant $(p=0.0005)$} & \multicolumn{3}{|c|}{$\mathrm{C}$ to 4 : significant $(\mathrm{p}=\mathbf{0 . 0 0 3})$} \\
\hline & \multirow{2}{*}{\multicolumn{3}{|c|}{$\begin{array}{l}C \text { to } P 1: \text { not significant }(p=0.14) \\
C \text { to } P 2 \text { : not significant }(p=0.82)\end{array}$}} & \multicolumn{3}{|c|}{ C to $P 1$ : not significant $(p=0.98)$} & \multicolumn{3}{|c|}{ C to $P 1$ : not significant $(p=0.38)$} \\
\hline & & & & \multirow{2}{*}{\multicolumn{3}{|c|}{$\begin{array}{l}C \text { to } P 2 \text { : not significant }(p=0.87) \\
C \text { to } P \text { 3: not significant }(p=0.79)\end{array}$}} & \multicolumn{3}{|c|}{$\begin{array}{l}C \text { to } P 2 \text { : not significant }(p=0.90) \\
\text { C to P3: not significant }(p=0.61)\end{array}$} \\
\hline & \multicolumn{3}{|c|}{$\mathrm{C}$ to $\mathrm{P}$ 3: not significant $(\mathrm{p}=0.45)$} & & & & C to P3: not & ignificant $(\mathrm{p}=$ & .61) \\
\hline & \multicolumn{3}{|c|}{ C to $P 4$ : not significant $(p=0.54)$} & \multicolumn{3}{|c|}{ C to $P 4$ : not significant $(\mathrm{p}=0.51)$} & \multicolumn{3}{|c|}{$\mathrm{C}$ to $\mathrm{P}$ 4: not significant $(\mathrm{p}=0.91)$} \\
\hline
\end{tabular}

Table 3 Colour Doppler sonographic results of haemodynamically relevant variables in the ophthalmic artery

\begin{tabular}{|c|c|c|c|c|c|c|c|c|c|}
\hline & \multicolumn{3}{|l|}{$\operatorname{Vmax}_{O A}$} & \multicolumn{3}{|l|}{$\operatorname{Vmin}_{O A}$} & \multicolumn{3}{|l|}{$P I_{O A}$} \\
\hline & Melanoma & Partner $(P)$ & Control (C) & Melanoma & Partner $(P)$ & Control (C) & Melanoma & Partner $(P)$ & Control $(C)$ \\
\hline Before therapy (1) & $0.32(0.11)$ & $0.33(0.11)$ & $0.34(0.1)$ & $0.1(0.05)$ & $0.10(0.05)$ & $0.09(0.02)$ & $1.38(0.25)$ & $1.58(0.38)$ & $1.58(0.27)$ \\
\hline After 6 months (2) & $0.3(0.13)$ & $0.36(0.11)$ & - & $0.08(0.05)$ & $0.10(0.05)$ & - & $1.56(0.43)$ & $1.54(0.35)$ & - \\
\hline After 12 months ( 3 ) & $0.3(0.09)$ & $0.34(0.08)$ & - & $0.08(0.05)$ & $0.10(0.04)$ & - & $1.49(0.44)$ & $1.40(0.37)$ & - \\
\hline After 24 months (4) & $0.36(0.20)$ & $0.34(0.08)$ & - & $0.1(0.06)$ & $0.09(0.04)$ & - & $1.54(0.3)$ & $1.50(0.41)$ & - \\
\hline Statistically significant & \multicolumn{3}{|c|}{$\mathrm{C}$ to 1 : not significant $(\mathrm{p}=0.59)$} & \multicolumn{3}{|c|}{ C to 1 : not significant $(p=0.37)$} & \multicolumn{3}{|c|}{$C$ to 1 : not significant $(p=0.07)$} \\
\hline in Wilcoxon's test & \multirow{2}{*}{\multicolumn{3}{|c|}{$\begin{array}{l}C \text { to } 2 \text { : not significant }(p=0.35) \\
C \text { to 3: not significant }(p=0.28)\end{array}$}} & \multicolumn{3}{|c|}{ C to 2 : not significant $(p=0.71)$} & \multicolumn{3}{|c|}{$\mathrm{C}$ to 2 : not significant $(\mathrm{p}=0.89)$} \\
\hline$(\mathrm{p}=<0.05)$ & & & & \multirow{2}{*}{\multicolumn{3}{|c|}{$\begin{array}{l}C \text { to } 3 \text { : not significant }(p=0.91) \\
C \text { to } 4 \text { : not significant }(p=0.43)\end{array}$}} & \multicolumn{3}{|c|}{ C to 3: not significant $(p=0.49)$} \\
\hline & \multicolumn{3}{|c|}{$C$ to 4 : not significant $(p=0.65)$} & & & & C to $4:$ not $\mathrm{s}$ & nnificant $(\mathrm{p}=0$ & \\
\hline & \multicolumn{3}{|c|}{ C to $P 1$ : not significant $(p=0.82)$} & \multicolumn{3}{|c|}{ C to $P 1$ : not significant $(p=0.79)$} & \multicolumn{3}{|c|}{ C to $P 1$ : not significant $(p=0.90)$} \\
\hline & \multicolumn{3}{|c|}{ C to P2: not significant $(\mathrm{p}=0.68)$} & \multicolumn{3}{|c|}{ C to P2: not significant $(\mathrm{p}=0.60)$} & \multicolumn{3}{|c|}{ C to $P 2$ : not significant $(p=0.96)$} \\
\hline & \multirow{2}{*}{\multicolumn{3}{|c|}{$\begin{array}{l}\text { C to P3: not significant }(p=0.90) \\
\text { C to P4: not significant }(p=0.91)\end{array}$}} & \multirow{2}{*}{\multicolumn{3}{|c|}{$\begin{array}{l}C \text { to } P \text { 3: not significant }(p=0.60) \\
C \text { to } P \text { : not significant }(p=0.54)\end{array}$}} & \multicolumn{3}{|c|}{$\begin{array}{l}C \text { to } P 3 \text { : not significant }(p=0.35) \\
C \text { to P4: not significant }(p=0.37)\end{array}$} \\
\hline & & & & & & & \multicolumn{3}{|c|}{ C to $P 4$ : not significant $(\mathrm{p}=0.37)$} \\
\hline
\end{tabular}

An alpha adjustment (Bonferroni-Holm) of the significance level was not suggested by our statistician because we performed a descriptive analysis only. An alpha adjustment would be helpful in an interference statistical analysis which would require more subjects.

\section{Results}

All patients except one were followed for 24 months. One patient died 19 months after radiotherapy as a result of multiple metastases.

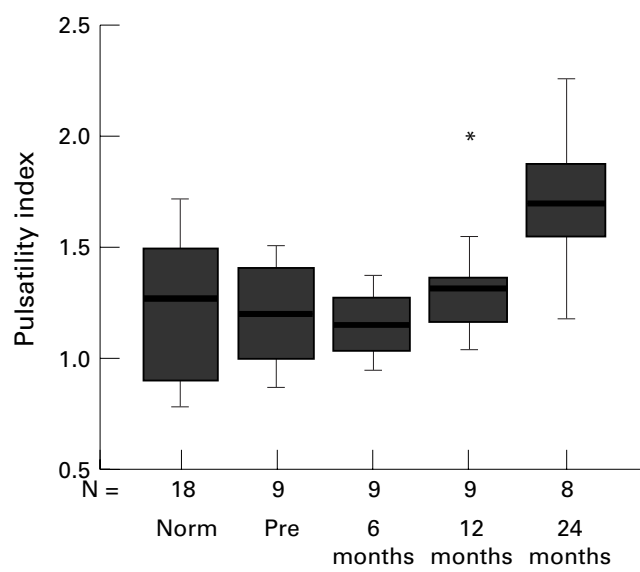

Figure 1 Pulsatility index in the short posterior ciliary arteries. Control (Norm), Pre = before therapy, 6 months after therapy, 12 months after therapy, and 24 months after therapy. $N$ is the number of individuals investigated. Box plots: summary plot based on median, quartiles, and extreme values. The box represents the interquartile range which contains $50 \%$ of the values. The whiskers are lines that extend from the box to the highest and lowest values, excluding outliers and extremes. A line across the box indicates the median. Extremes: cases with values more than three box lengths from the upper or lower edge of the box. The box length is the interquartile range. Outliers: cases with values between 1.5 and 3 box lengths from the upper or lower edge of the box.
All patients in this series demonstrated local tumour control with reduction of tumour thickness from $6.8 \mathrm{~mm}$ initially to 4.6 (SD 2.3) $\mathrm{mm}$ (range $2.0-9.4 \mathrm{~mm}$ ).

Blood flow velocity was significantly reduced in the CRA at all times of measurement, comparing melanoma eyes with controls (see Table 2).

No statistically significant differences were found when comparing blood flow velocities in the OA and PCA of the melanoma eyes with the controls at baseline, at the 6 months, and 12 months of follow up. At the 24 month check, blood flow velocity in the OA still showed no difference. However, at 24 months the end diastolic blood flow velocity (Vmin) of the PCA of the melanoma eyes was significantly reduced whereas the systolic blood flow velocity was not.

No statistically significant differences were found when comparing PI values of the OA, the PCA, and the CRA of the melanoma eyes with the controls at baseline, at 6 months, and 12 months of follow up. At the 24 month check, PI in the OA still indicating no difference whereas the PI of the PCA and CRA of the melanoma eyes was significantly increased (Tables 1-3, Figs 1 and 2).

Three eyes developed retinopathy and were treated with argon laser, whereas opticopathy was found in two cases.

\section{Discussion}

An effective vasculature is essential for tumour growth. Many authors report the use of colour Doppler to measure tumour blood flow velocity in malignant melanomas of the uvea and describe frequency shifts after radiotherapy. ${ }^{10-12} 16$ 


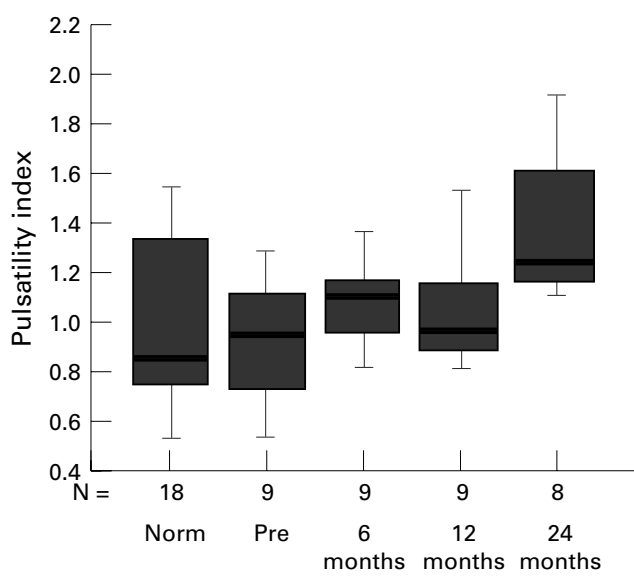

Figure 2 Pulsatility index in the central retinal artery. Control (Norm), Pre = before therapy, 6 months after therapy, 12 months after therapy, and 24 months after therapy. $N$ is the number of individuals investigated. Box plots: summary plot based on median, quartiles, and extreme values. The box represents the interquartile range which contains $50 \%$ of the values. The whiskers are lines that extend from the box to the highest and lowest values, excluding outliers and extremes. A line across the box indicates the median. Extremes: cases with values more than three box lengths from the upper or lower edge of the box. The box length is the interquartile range. Outliers: cases with values between 1.5 and 3 box lengths from the upper or lower edge of the box.

Lieb et al found abnormal Doppler shifts within neoplastic lesions and lower Doppler shifts in choroidal melanomas after radiation therapy. ${ }^{10}$ Gutthof et al concluded that colour Doppler allows a rapid overview of tumour vasculature and facilitates the study of individual vessels. ${ }^{11}$ Wolff-Kormann et al reported a decrease in peak systolic frequency in choroidal melanomas within the tumour and in the tumour base after episcleral brachytherapy. ${ }^{12}$ Cennamo et al suggested the use of an ultrasound contrast agent to enhance the Doppler signal in evaluating the effectiveness of radiotherapy in malignant tumours. ${ }^{16}$ Yang et al reported a higher pulsatile ocular blood flow in eyes with untreated choroidal melanoma than in unaffected eyes when using an ocular blood flow tonograph. They concluded that in the presence of a melanoma there is a higher blood flow or a global increase in choroidal blood flow. ${ }^{18}$

When performing Doppler studies for tumour vessels or tumour base vessels, some difficulties are encountered: the location of blood flow velocity measurement in the tumour vessel lacks reproducibility; the direction of blood flow is often not identifiable; the angle between the vessel and the transducer often exceeds $60^{\circ}$, thus making the results inexact.

After therapy, a change in blood flow is normally found $\mathrm{d}^{10-12}$ and the disappearance of tumour vessels is desirable. In follow up studies with Doppler, however, it is difficult to obtain measurements from the same spot within the tumour. If many vessels supply the tumour, it is a problem to take accurate measurements from the same vessel for each investigation.

Contrary to previous studies, we investigated blood flow velocities and resistance variables at defined and reproducible locations of ocular blood supply. The video documentation provided in the system helped to find the same points of measurement at each follow up examination. Tumour vessels were avoided.

We found no statistically significant changes of blood flow velocities and vessel resistance variables (PI, RI) in the OA and PCA of melanoma eyes, comparing the baseline results with the 6 month and 12 month follow up examinations. Blood flow velocity and vessel resistance variables stayed unaltered in the $\mathrm{OA}$ until the 2 year follow up measurements. However, blood flow velocity and vascular resistance in the short posterior arteries of melanoma eyes were significantly altered at the 2 year follow up. Furthermore, there were no significant differences in comparing blood flow velocities and vessel resistance variables (PI, $\mathrm{RI}$ ) in the OA and PCA of the melanoma eyes with the contralateral eye or with the controls at the 6 month and 12 month follow up. There were also no significant changes in blood flow velocity and vessel resistance variables in the OA at the 24 month measurement. But, blood flow velocity and vascular resistance in the short posterior arteries were significantly altered at the 2 year follow up.

Blood flow velocity was significantly reduced in the CRA at all times of measurement in melanoma eyes compared with healthy controls, whereas RI and PI showed a significant increase compared with controls but not before the 24 month check. At that time, blood flow velocity in the CRA was reduced and vessel resistance variables of the small vessels were increased.

The reduced blood flow velocity presented initially might be a steal effect from the CRA towards the tumour area. High dose stereotactic radiotherapy with the Leksell gamma knife and 41-66.5 Gy total marginal dose in two fractions influences the ocular blood supply by increasing vascular resistance of the small arteries within 2 years after treatment. We assume that a slowly developing radiogenic vasculopathy of the small orbital vessels is the cause of these increased vessel resistance variables.

The high number of side effects (three eyes with retinopathy, two eyes with opticopathy) was related mainly to the size and the location of the melanoma, being unsuitable for ruthenium-106 brachytherapy. Only melanomas at the posterior pole in vicinity of the fovea or the optic nerve $(<3 \mathrm{~mm}$ distance) or tumours with a thickness exceeding $7 \mathrm{~mm}$ were treated with gamma knife stereotactic radiosurgery.

We considered ciliary body tumours exceeding $7 \mathrm{~mm}$ thickness and $>3$ clock hours of circumferential extension as unsuitable for ruthenium-106 brachytherapy or surgical extension. In these cases, gamma knife irradiation was performed as an alternative to primary enucleation.

A melanoma eye suffers from decreasing blood supply in two ways-initially due to the steal effect of blood towards the tumour and, in the long run, as a consequence of radiation. 
Our findings are that there are decreased blood flow and increased vascular resistance in the central retinal artery and the short posterior ciliary arteries after gamma knife radiosurgery. We conclude that gamma knife irradiation influences the normal vascular structures. These findings may be related to the radiosensitivity of the small vessels as well as to total dose and fractionation. Whether decreased blood flow has a prognostic value for the patient requires further investigation.

1 Mitchell DG. Colour Doppler imaging: principles, limitations and artefacts. Radiology 1990;177:1-10.

2 Powis RL. Colour flow imaging:understanding its science and technology. F Diagn Med Sonogr 1988;4:234-45.

3 Maslak SH, Freund JG. Colour Doppler instrumentation. In Lanzer P, ed. Vascular imaging by colour Doppler and mag netic resonance. Berlin, Heidelberg: Springer, 1991:87-122.

4 Deane CR, Forsberg F, Thomas N, et al. Accuracy of colour Doppler ultrasound velocity measurements in small vessels. f Biomed Eng 1991;13:249-54.

5 Pourcelot L. Clinical applications of transcutaneous Doppler examinations. In: Perenneau P, ed. Velocimetrie ultrasonore Doppler. Paris: Inserm, 1975:213-40.

6 Gosling RC, King DH. Arterial assessment by Doppler shift ultrasound. Proc R Soc Med 1974;67:447-9.

7 Erickson SJ, Hendrix LE, Massaro BM, et al. Colour Doppler flow imaging of the normal and abnormal orbit. Radiology 1989;173:511-6.
8 Giovagnorio F, Ouaranta L, Bucci MG. Colour Doppler

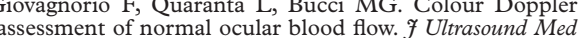
1993;12:473-7.

9 Lieb WE, Flaharty PM, Ho AC, et al. Colour Doppler imaging of the eye and orbit. A synopsis of a 400 case experience. Acta Ophthalmol 1992;204 (Suppl):50-4

10 Lieb WE, Shields JA, Cohen SM, et al. Colour Doppler maging in the management of intraocular tumours.Ophthalmology 1990;97:1660-4.

11 Guthoff RF, Berger RW, Winkler P, et al. Doppler ultrasonography of malignant melanomas of the uvea. Arch Ophthalmol 1991;109:537-41.

12 Wolff-Kormann PG, Kormann BA, Riedel KG, et al Quantitative colour Doppler imaging in untreated and irradiated choroidal melanoma. Invest Ophthalmol Vis Sci 1992:33: 1928-33.

13 Zehetmayer M, Menapace R, Kitz K, et al. Stereotactic irradiation of uveal melanoma with the Leksell Gamma Unit. In: Wiegel T, Bornfeld N, Foerster MH, Hinkelbein W, eds. Radiotherapy of ocular disease. Front Radiat Ther Oncol. Radiotherapy of ocular disease.

$14 \mathrm{Wu}$ A. Physics and dosimetry of the gamma knife. Neurosurg Clin N Am 1992;3:35-50.

5 Zehetmayer M, Menapace R, Kitz K, et al. Suction attachment for stereotactic radiosurgery of intraocular malignancies. Ophthalmologia 1994;208:119-21.

16 Cennamo G, Rosa N, Vallone GF, et al. First experience with a new echographic contrast agent. $\mathrm{Br} \mathcal{F}$ Ophthalmol 1994;78:823-6.

17 Quaranta L, Harris A, Donato F, et al. Colour Doppler imaging of ophthalmic artery blood flow velocity: a study of repeatability and agreement. Ophthalmology 1997;104:6538 .

18 Yang YC, Kent D, Fenerty CH, et al. Pulsatile ocular blood flow in eyes with untreated choroidal melanoma. Eye 1997; $11: 331-4$ 